\title{
Utilizing local anti-cancer treatment and online medical service during the COVID-19 pandemic
}

\author{
Qi Wang, Zhouqiao Wu, Jinyao Shi, Kan Xue, Ziyu Li, Jiafu Ji
}

Received 05 February 2021 accepted 10 March 2021
Carcinogenesis and Translational Research (Ministry of Education), Gastrointestinal Cancer Center, Peking University Cancer Hospital \& Institute, No.52 Fucheng Road, Beijing, 100142, China

*Corresponding author Jiafu Ji, E-mail: jijiafu@hsc.pku.edu.cn
Since the first outbreak of the coronavirus disease (COVID-19) in the winter of 2019, this pandemic has resulted in significant global social and economic disruption, as well as global public health and medical crises[1]. Climate, weather conditions, and even latitude have now been acknowledged to be directly associated with COVID-19 outbreaks[2-4]. It was reported that $60 \%$ of the confirmed cases of COVID-19 occurred in areas where the air temperature ranged from $5^{\circ} \mathrm{C}$ to $15^{\circ} \mathrm{C}$ [4]. These earlier research results strongly implied that the COVID-19 pandemic might spread cyclically and outbreaks might recur in large cities in the mid-latitudes (for example, the northern part of China) in autumn 2020[4], which has now been confirmed by the third wave of the pandemic. With quarantines and other restrictive legislations and control measures being put in action to contain the spread of the disease[5], the pandemic has also manifested additional obstacles in the treatment of other diseases. For example, a median dose delay of 14 (6-20) days was found in patients who received one cycle of gastric chemotherapy in our department from the first outbreak till the spring festival of $2020(n=79)$.

Many cancer patients intend to seek treatments in top-tier public hospitals in big cities. The majority $(62 / 79,78.5 \%)$ of our patients come from other provinces throughout the country. Concerns were raised that the pandemic and the subsequent travel restrictions might postpone patients' planned trips to the hospital. Such a circumstance prompted the China gastrointestinal cancer surgical union (CGCSU) to update the clinical information of the local hospitals during the epidemic[6], because of which 55 of our patients switched their chemotherapy in local community health care providers.

Due to various countermeasures being applied to minimizing the impact of COVID-19 on cancer patients, the majority of our patients $(77.2 \%, 61 / 79)$ received chemotherapy within 3 weeks (one cycle period) after the planned date. Among them 23 had a dose delay $\leq 7$ days, and most of them $(86.0 \%, 20 / 23)$ were from the group of patients treated locally, which turned out to be an independent protective factor (OR: $0.159 ; 95 \% \mathrm{Cl}: 0.039-0.645 ; P$ $=0.010$ ).

The great potential and capacity of health care services provided to cancer patients by local organizations was unforeseen, because inequality of health care availability has persisted in China[7]. Prior to pandemic, one might assume that epidemic-induced traffic restrictions would throw the existing inequalities of China's health care system into a sharp relief. Contrarily, our data showed that most patients, even those living in second- and third-tier provinces had easy access to complex medical treatments via local health care providers. The travel-restrictions during epidemics have presented local health care providers with the opportunity of shouldering unprecedented health care responsibilities. And with cross-institutional cooperation, community care support services organizations and medical facilities could help relieve the burden of major hospitals in large/mega cities in times of emergency.

To minimize the influence of traffic restrictions, our hospital has provided online service for revisiting patients since July 2020 when new confirmed COVID-19 cases started to raise again. Online graphic consultation and video call consultation, and even prescription of examinations and medicines can be easily accessed via the hospital app on a mobile phone. Only when receiving treatment or examination, do patients need to come to the hospital. By January 2021, the online service system has served 45772 patients, issued 119185 examination and test application forms and 13872 drug prescriptions. This partly overcomes the obstacle of the travel restrictions during epidemics, providing a continuous treatment for our patients at best.

Our data and experience indicate that integrating all available health resources including local and remote hospitals and online medical service is a feasible strategy for providing uninterrupted regular medical services while responding to devastating public 
health emergency. It is anticipated, therefore, that making full use of local hospitals and online medical services should also be of great benefits and advantages in times of non-emergencies, which warrants serious consideration and discussion in the policymaking process to address the inequalities issue existing in China's health care system.

\section{Ethical approval and informed consent}

This study was approved by the ethical committee of Peking University Cancer Hospital. Patients or the public were not involved in the design, or conduct, or reporting, or dissemination plans of our research.

\section{Conflicts of interest}

We declare no competing interests. This study was approved by the ethical committee of Peking University Cancer Hospital. We thank all participating centers of CGCSU for providing updated information and continuous service to cancer patients during the pandemic.

\section{Acknowledgments}

This work was supported by the Summit Talent Plan (to JJ, No. DFL20181103) and Young Scholar Program of Beijing Hospitals Authority (to ZW, No. QMS20191103).

\section{References}

[1] Li Q, Guan X, Wu P, et al. Early transmission dynamics in Wuhan, China, of novel coronavirus-infected pneumonia. New England Journal of Medicine, 2020; 382: 1199-1207.

[2] Baker R E, Yang W, Vecchi G A, et al. Susceptible supply limits the role of climate in the early SARS-CoV-2 pandemic. Science (New York, NY), 2020; 369(6501): 315-319.

[3] Suthar S, Das S, Nagpure A, et al. Epidemiology and diagnosis, environmental resources quality and socio-economic perspectives for COVID-19 pandemic. Journal of environmental management 2021; 280 : 111700.

[4] Huang Z, Huang J, Gu Q, et al. Optimal temperature zone for the dispersal of COVID-19. The Science of the total environment, 2020; 736 : 139487.

[5] Chen S, Yang J, Yang W, et al. COVID-19 control in China during mass population movements at New Year. The Lancet, 2020; 395: 764766.

[6] China gastrointestinal cancer surgical union. Consultation Information for Gastrointestinal Cancer Patients During the COVID-19 Outbreak. https://mp.weixin.qq.com/s/zORiGHeETfeTZr8AfmctbQ (accessed Feb. 29 2020).

[7] Anand S, Fan V Y, Zhang J, et al. China's human resources for health: quantity, quality, and distribution. Lancet, 2008; 372(9651): 1774-1781. 